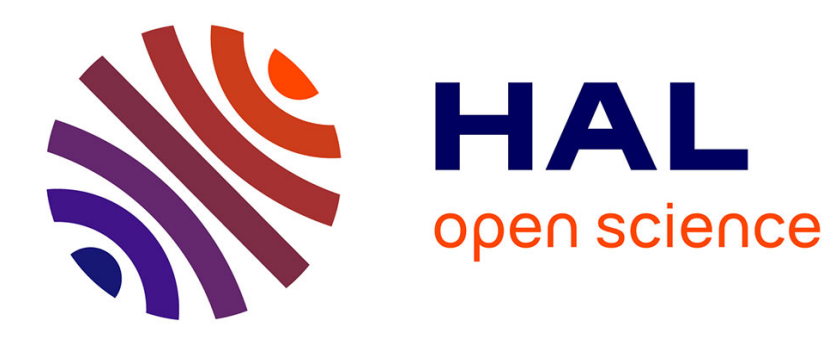

\title{
Calcul de propagation des vibrations dans un rail libre
}

L. Gavric

\section{To cite this version:}

L. Gavric. Calcul de propagation des vibrations dans un rail libre. Journal de Physique IV Proceedings, 1994, 04 (C5), pp.C5-73-C5-76. 10.1051/jp4:1994508 . jpa-00252770

\section{HAL Id: jpa-00252770 https://hal.science/jpa-00252770}

Submitted on 1 Jan 1994

HAL is a multi-disciplinary open access archive for the deposit and dissemination of scientific research documents, whether they are published or not. The documents may come from teaching and research institutions in France or abroad, or from public or private research centers.
L'archive ouverte pluridisciplinaire HAL, est destinée au dépôt et à la diffusion de documents scientifiques de niveau recherche, publiés ou non, émanant des établissements d'enseignement et de recherche français ou étrangers, des laboratoires publics ou privés. 


\title{
Calcul de propagation des vibrations dans un rail libre
}

\author{
L. GAVRIĆ
}

CETIM, Département Acoustique Industrielle, 52 Avenue Felix Louat, BP. 67, 60304 Senlis, France

\begin{abstract}
RESUME: A waveguide finite element theory is applied to the propagation pheneomena in rails. In the first part of the paper the waveguide finite element computation technique is briefly presented. The propagative wavenumbers and corresponding modes of a rail are computed as function of the exctation frequency. The results of finite element computation are compared with those obtained by the simple Euler-Bernoulli beam theory for the flexion. The sound radiation and the distribution of the energy transmitted through the cross-section by the computed propagative modes are analysed. The present analysis gives the valuable informations on the participation of different propagative modes to vibro-acustical response of rails.
\end{abstract}

\section{INTRODUCTION}

Les premiers calculs de propagation des vibrations dans les rails ont été faits en utilisant la théorie des poutres. Le rail est modélisé comme une poutre infinie selon la théorie d'EulerBernoulli ou selon la théorie de Timoshenko. Cette denière prend en compte les effets de cissaillement et d'inertie de rotation qui ne figurent pas dans la théorie simplifiée d'Euler-Bernoulli.

Les modèles basés sur la théorie des poutres entrainent une imprécision qui est due principalement au fait que:

- couplage des vibrations de "torsion" et de "flexion horizontale" n'est pas pris en compte. En effet, la section droite $d^{\prime}$ un rail a un axe de symétrie vertical, qui découple les "vibrations verticales" des autres types de vibrations. Au contraire, lors des vibrations de type "flexion horizontale" le rail subit simultanément des dêplacements caractéristiques pour la "torsion" et vice-versa.

- le deuxième effet qui limite fortement l'utilisation des modèles simples pour le calcul du comportement dynamique des rails est la déformation de la section droite qui est particulierement marquée pour une excitation dans le domaine moyennes et hautes fréquences.

- pour des fréquences d'excitation élevées la présence d'autres types d'ondes que ceux définis par la théorie des poutres est observée

Pour améliorer les techniques existantes une nouvelle approche du calcul previsionel du comportement dynamique du rail a été développée. Le calcul permet une caractérisation vibroacoustique pour une gamme de fréquences comprises entre $0-6000[\mathrm{~Hz}]$. L'idée directrice de ce nouveau concept est de considérer le rail comme un guide d'ondes dont le comportement dynamique est décrit par un ensemble d'ondes élastiques propres qui sont à l'origine du champ acoustique créé autour du rail. Le calcul numérique des courbes de dispersion est basé sur la méthode des éléments finis spécialement développée pour l'analyse de guides d'ondes. 


\section{PROPAGATION DES VIBRATIONS DANS DES GUIDES D'ONDES}

La propagation libre dans un guide d'ondes avec la section droite dans le plan $x-y$ et avec la direction de propagation parallèle avec l'axe $z$ est décrite par le champ de déplacements suivant:

$$
\tilde{u}_{i}(x, y, z, t)=\tilde{u}_{i}(y, z) e^{-j \tilde{\kappa} x} e^{j \omega t} e^{j \theta_{i}} \quad \theta_{x}=\theta_{y}=0 ; \theta_{z}=\frac{\pi}{2}
$$

où les quantités complexes sont désignées par $(\tilde{\ldots}), j=\sqrt{-1}$ représente l'unité imaginaire et l'indice les coordonées $i=x, y, z$. Un tel champ de déplacements implique qu'un point du guide d'ondes, défini par les coordonnées $x, y$ et $z$, subit le mouvement harmonique avec la pulsation $\omega$. Les ondes se propagent dans la direction $z$ avec le nombre d'onde $\tilde{\kappa}$, tandis que la fonction spatialle $\tilde{u}_{i}(x, y)$ décrit le mouvement de la section droite pendant le passage des ondes, [1]. Dans ce cas les déformations et les contraintes deviennent:

$$
\tilde{\varepsilon}_{i j}(x, y, z, t)=\tilde{\varepsilon}_{i j}(x, y, \tilde{\kappa}) e^{-j \tilde{\kappa} z} e^{j \omega t} \quad ; \quad \tilde{\sigma}_{i j}(x, y, z, t)=\tilde{\sigma}_{i j}(y, z, \tilde{\kappa}) e^{-j \tilde{\kappa} x} e^{j \omega t}
$$

Ces deux tenseurs sont reliés entre eux par la loi du comportement $\tilde{\sigma}_{i j}=E_{i j k l} \tilde{\varepsilon}_{k l}$, ou $E_{i j k l}$ est le tenseur d'élasticité, [2]. En utilisant les quantités définies précédemment, le principe des travaux virtuels pour la propagation libre dans le guide d'ondes devient fonction des deux variables spatiales $x$ et $y$ et du nombre d'onde $\tilde{\kappa}$ :

$$
\int_{\Omega}\left[\tilde{\sigma}_{i j}(x, y, \tilde{\kappa}) \delta \tilde{\varepsilon}_{i j}^{*}(x, y, \tilde{\kappa})-\omega^{2} \rho(x, y) \tilde{u}_{i}(x, y) \delta \tilde{u}_{i}^{*}(x, y)\right] d x d y=0
$$

où $\rho(x, y)$ est la masse volumique. Les quantités virtuelles sont notées par $\delta$ tandis que $(\ldots)^{*}$ désigne le conjugué complexe. Il convient de remarquer que l'intégration est effectuée dans le domaine spatial à deux dimensions qui définissent la section droite $\Omega=\Omega(x, y)$, [3]. Le premier terme de l'Eq.(3), qui dépend du nombre d'onde $\tilde{\kappa}$, représente l'énergie potentielle (énergie de déformation élastique), tandis que le deuxième terme correspond à l'énergie cinétique. Les équations des éléments finis sont obtenues par la discrétisation du domaine $\Omega$ et en approximant le champ de déplacement dans chaque élément fini par ses fonctions de forme. Pour un vecteur arbitraire de déplacements virtuels, la procédure aboutit au système linéaire d'équations agébriques:

$$
\left(\tilde{\kappa}^{2}\left[K_{2}\right]+\tilde{\kappa}\left[K_{1}\right]+\left[K_{0}\right]-\omega^{2}[M]\right)\{\tilde{U}\}=0
$$

où $\left[K_{2}\right],\left[K_{1}\right]$ et $\left[K_{0}\right]$ sont les matrices de raideur, $[M]$ est la matrice de masse et $\{\tilde{U}\}$ est le vecteur de déplacements. Le problème numérique consiste à trouver les scalaires $\tilde{\kappa}_{1}, \tilde{\kappa}_{2}, \ldots \tilde{\kappa}_{n}, \ldots$ et les vecteurs $\left\{\tilde{U}_{1}\right\},\left\{\tilde{U}_{2}\right\}, \ldots\left\{\tilde{U}_{n}\right\}, \ldots$ qui satisfassent l'équation précédente pour une pulsation donnée $\omega$. Si le paramètre scalaire calculé $\kappa_{n}$ est réél le vecteur correspondant $\left\{U_{n}\right\}$ l'est aussi. Dans ce cas, les quantités obtenues représentent le nombre d'onde propagatif $\kappa_{n}$ et le mode propagatif de la section droite $\left\{U_{n}\right\}$. Les solutions complexes pour les nombres d'ondes et les modes correspondent aux ondes évanescentes, qui généralement ne propagent pas d'énergie. La distribution de l'énergie mécanique transportée par une onde propagative peut être évaluée en utilisant l'intensité vibratoire active dans la direction de propagation. Pour le champ de déplacement défini par Eq.(1), cette intensité prend la forme suivante, [4]:

$$
I_{z}=\frac{\omega}{2}\left[\sigma_{z z} u_{z}-\tau_{x z} u_{x}-\tau_{y z} u_{y}\right]
$$

où $\sigma_{z z}, \tau_{x z}, \tau_{y z}$ et $u_{x}, u_{y}, u_{z}$ représentent les amplitudes réelles des contraintes et des déplacements de l'onde propagative considerée. Ces grandeurs sont évaluées aux centres des éléments en utilisant les fonctions de formes, leurs dérivées et la loi de comportement. 


\section{THEORIE DES GUIDES D'ONDES APLIQUEE A UN RAIL}

\subsection{Courbes de dispersion dans la structure d'un rail}

Le calcul des nombres d'ondes et les modes de la section droite du rail UIC 861-3 est effectué pour la gamme fréquentielle 0-6000 [Hz] en utilisant la méthode décrite précédemment. Sur les courbes de dispersion de deux ondes, deux modes correspondant sont présentés en fonction de la fréquence d'excitation, Fig.(1).

FLEXION VERTICALE

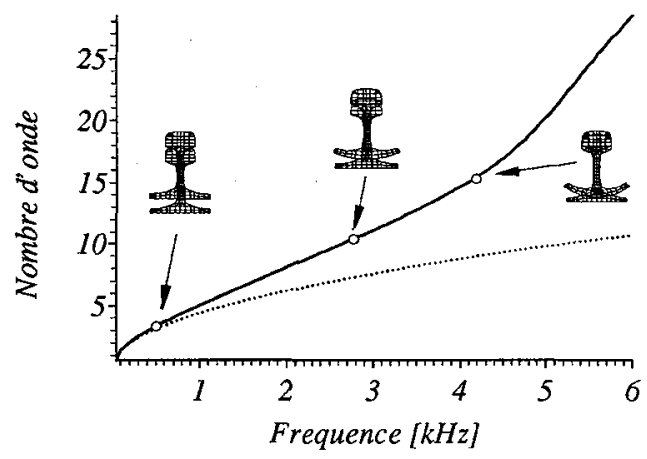

FLEXION HORIZONTALE

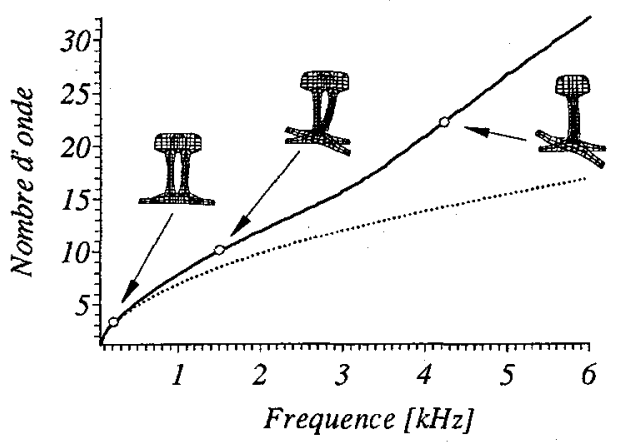

Fig. 1. Courbes de dispersion de deux ondes propagatives et modes de la section droite

Sur ces courbes, les résultats du calcul numérique (trait continu) sont superposés avec les résultats obtenus en utilisant la théorie des poutres Euler-Bernoulli (pointillé). La comparaison des courbes de dispersion montre que la théorie d'Euler-Bernoulli n'est valable que pour les fréquences basses.

\subsection{Propagation d'énergie dans la structure d'un rail}

Pour les fréquences élevées d'autres types d'ondes propagent de l'énergie mécanique. Au dessus de $\sim 1300[\mathrm{~Hz}]$ une onde supplémentaire devient propagative. Une déformation du type "flexion de section droite" corresponde au mode de l'onde, Fig.(2.c).

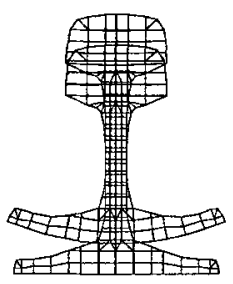

(a)

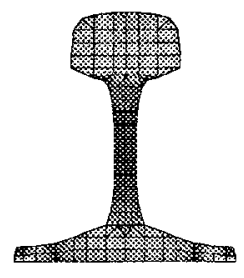

(b)

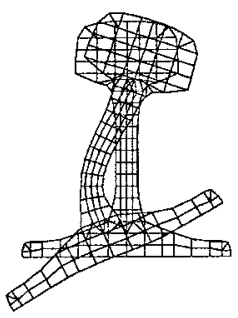

(c)

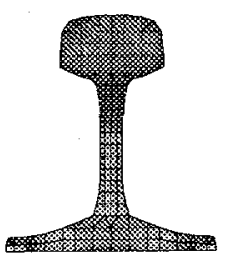

(d)

Fig. 2. Modes et distribution de l'énergie: $a, b)$ flexion verticale ; $c$,d) flexion de section

Pour la fréquence d'excitation 2800 [Hz], où les deux ondes ont les longueurs d'ondes comparables $\sim 0.6$ [m], les énergies propagées par la "flexion de section droite" et par la "flexion verticale" sont 
évaluées par l'intégration de l'intensité sur la section droite du rail. Le calcul montre que l'onde de type "flexion de section droite" propage presque deux fois plus d'énergie que la "flexion verticale".

\subsection{Rayonnement des modes propagatifs}

Les vitesses vibratoires des modes servent comme données pour le calcul du rayonnement du rail. En suite, l'analyse des résultats du calcul permet d'évaluer l'importance relative des différents modes qui contribuent à la création du champ acoustique autour du rail.

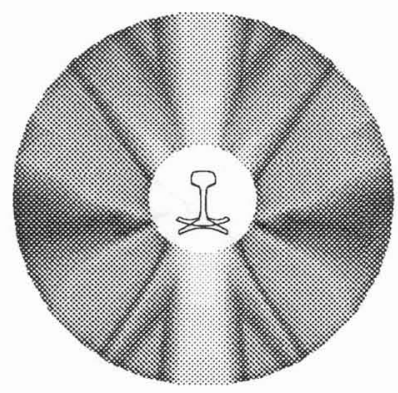

(a)

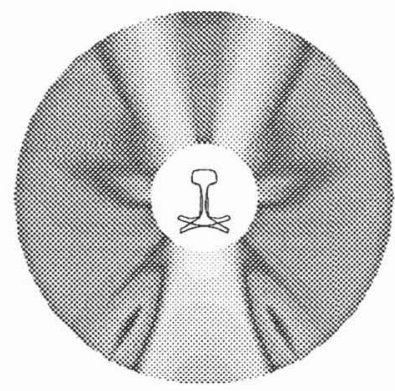

(b)

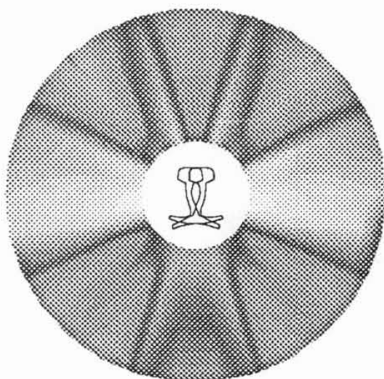

(c)

Fig. 3. Rayonnement des modes propagatifs - la pression acoustique quadratique

Les champs acoustiques créés par trois types d'ondes propagatives sont présentés en Fig.(3). Les modes correspondent aux ondes de type "flexion verticale" - Fig.(3.a), "flexion horizontale" Fig.(3.b) et "torsion" - Fig.(3.c) pour la fréquence d'excitation de 4200 [Hz]. Les resultats du calcul des champs acoustiques sont obtenus en utilisant la méthode des équations intégrales (collocation).

\section{CONCLUSIONS}

Une nouvelle approche du calcul du comportement dynamique de la structure d'un rail utilisant la méthode des éléments finis a montré qu'il est nécessaire de prendre en compte la déformation de la section droite, en particulaire dans le domaine fréquentielle au-delà de 1300 [Hz]. Ce concept, récemment développé et mis au point, permet la prévision précise du comportement vibro-acoustique de la structure du rail pour toute la gamme fréquentielle d'intèrêt, $0-5000[\mathrm{~Hz}]$.

- Les calcules sont effectués par la chaine des logiciels "GOD - Guides des Ondes Déformables" devéloppée au CETIM.

\section{R E F E R E N C E S}

[1] - KOLSKY H., Stress waves in solids ( New York: Dover Publications Inc., 1963).

[2] - DIEULESAINT E. \& ROYER D., Ondes élastiques dans les structures,

(Paris: Masson et $\left.C^{i e}, 1974\right)$.

[3] - GAVRIĆ L., Journal of Sound and Vibrations - in print for February 1994

Finite element computation of dispersion properties of thin-walled waveguides.

[4] - GAVRIĆ L., "Structural intensity in waveguides with deformable cross-section", 4-ème Congrès international sur l'intensimétrie, Senlis France, 31 août - 2 septembre,

(CETIM, 1993) pp.263-270. 